\title{
AJCC Tumor Grade
}

National Cancer Institute

\section{Source}

National Cancer Institute. A/CC Tumor Grade. NCI Thesaurus. Code C135461.

An assessment of the cellular differentiation of a tumor as per the American Joint Committee on Cancer (AJCC) tumor grading scale. 